\title{
A CHOICE THEORETIC FOUNDATION FOR SAATY'S EIGENVECTOR METHOD IN AHP
}

\author{
Saburo Saito \\ Faculty of Economics, Fukuoka University \\ 8-19-1 Nanakuma, Jonan-ku, Fukuoka, JAPAN 814-0180 \\ saito@fukuoka-u.ac.jp
}

\begin{abstract}
While Saaty's eigenvector method constitutes the founding stone for AHP, its validation still leaves an open problem: Why is the principal eigenvector of positive reciprocal matrix of pairwise comparison relevant for the ratio scale of the objects compared? This paper establishes a choice theoretic foundation for this open problem. The binary choice probability problem for given $n$ objects is an inverse problem to ...nd a probability distribution over $n$ ! rankings that induces given $\frac{n\left(n_{j} 1\right)}{2}$ binary choice probabilities. This paper reformulates Saaty's eigenvector method as a binary choice probability problem and shows that a certain equivalence exists between the binary choice probability problem and Saaty's method in the sense that the solution for the binary choice probability problem exactly reproduces the Saaty's eigenvector for $n=3$. This result gives a choice theoretic validation for Saaty's eigenvector method.
\end{abstract}

\section{Introduction}

While Saaty's eigenvector method constitutes the founding stone for AHP (Analytic Hierarchy Process), its validation still leaves an open problem: Why is the principal eigenvector of positive reciprocal matrix of pairwise comparison relevant for the ratio scale of the objects compared? This paper addresses this open problem and establishes a choice theoretic foundation for Saaty's eigenvector method.

The key idea in this paper is the 1-projection modeling for the binary choice probability problem and its application to characterizing Saaty's eigenvector method. In general, the I-projection problem is a convex program to ...nd a probability distribution that minimizes Kullback-Leibler information among the ones that satisfy some convex constraint. The solution for the I-projection problem is called the I-projection onto the convex constraint. The statistical method formulated as the Iprojection problem is referred to as I-projection modeling. (Saito (1998)) The I-projection modeling ...nds many applications in various ...elds such as economics, transportation science, statistics and so on. As for theoretical argument, the 1-projection problem is closely related to information theory, maximum entropy or minimum information principle, maximum likelihood estimation, inverse problem, and large deviation theory. Quite interesting is its relationship to the linear statistical inverse problem to choose the best probability distribution among the ones satisfying linear constraints. Under some axioms, the I-projection problem is derived logically as the best for obtaining the solution for the linear inverse problem. (Shore, Johnson (1980), Csiszar (1991))

Binary choice probabilities generically have the character of pairwise comparison. They are de...ned as $f p_{i j} g$ for $i \xi j$ such that $p_{i j}+p_{j i}=1$. Note that a positive reciprocal outcome matrix $f a_{i j} g$ can be transformed into binary choice probabilities $f p_{i j} g$ with one-to-one by $p_{i j}=\frac{a_{11}}{a_{1 j}+1}$, or equivalently, $a_{i j}=\frac{P_{13}}{P_{31}}$. Traditionally, the binary choice probability problem for $n$ objects has been known as a problem to ...nd the condition of when given $\frac{n\left(n_{j} 1\right)}{2}$ binary choice probabilities can be induced by some probability distribution over $n$ ! rankings, i.e., all linear orders of $n$ objects. However, as will be seen later, whether some probability distribution induces given binary choice probabilities can be expressed as linear equality constraints imposed on the probability distribution so that we formulate the binary choice probability problem as an inverse problem to ...nd the best probability distribution over $n !$ rankings that satis...es $\frac{n\left(n_{1} 1\right)}{2}$ binary choice probability constraints. 
This inverse problem formulation for binary choice probability problem makes possible some curious probabilistic argument and our use of 1-projection modeling for the solution method.

Based on these observations, this paper applies the I-projection modeling for the binary choice probability problem to the characterization of Saaty's eigenvector method to tackle the above open problem. I have demonstrated that a certain equivalence exists between a binary choice probability problem and Saaty's eigenvector method in the sense that the solution for the binary choice probability problem exactly reproduces the Saaty's eigenvector for $n=3$. More speci...cally, if the residual matrix of Saaty's method is transformed into binary choice probabilities, the Iprojection for this residual binary choice problem becomes "indiderent" to all three objects with respect to the probability how each object is ranked among three. In fact, the probability that each object is selected as the top becomes identical for all three objects.

Here the residual matrix is the elementwise division of an outcome matrix by the consistent matrix constructed by the Saaty's eigenvector. It is well known that the residual matrix becomes a scalar multiple of stochastic reciprocal matrix and its right principal eigenvector is 1, all of which components are 1's. (Saaty (1980), Vargas (1983)) This fact has been used for validating Saaty's eigenvector method since no information is remained in the residual matrix from the viewpoint of eigenvector. In the same way, the above result of this paper implies that no "partial" information is contained in the residual matrix from the binary choice perspective, either. This is because the 1-projection solution for the residual makes no partial contribution to the probability how each object is ranked among three. Hence this gives another validation for Saaty's eigenvector method.

The interpretation of inconsistency for pairwise comparison need not be limited to misjudgment by respondent. In fact, since its solution of probability distribution over $n$ ! rankings is a convex mixture of $n$ ! distinct preferences, we see that the I-projection modeling for binary choice probability problem regards the inconsistency as arising from the mixture of $n !$ distinct preferences. Thus it can be interpreted as a theoretical method to decompose aggregated preferences of binary choice probabilities into a convex mixture of distinct consistent preferences. (Saito $(1989,1998)$ ) It should be noticed that the more detailed analysis of inconsistent pairwise comparison becomes possible for I-projection modeling than Saaty's eigenvector method.

\section{Binary choice probability problem}

Let $N$ denote a set of alternatives. Consider a binary relation $R$ on $N \in N$. Denote $x R y$ if $(x ; y) 2 R \quad 1 / 2 N \in N$ with understanding that $x$ is "preferred to" $y$. Assume that the binary relation $\mathrm{R}$ is a linear order, i.e., irrełexive, transitive, and complete. Under a linear order $\mathrm{R}$, all of the alternatives can be ordered from the most preferred to the least. Thus the set $\mathrm{S}$ of all distinct linear orders on $\mathrm{N} \perp \mathrm{N}$ consists of $\mathrm{jNj}$ ! rankings. Consider the probability distribution $\mathrm{X}$ on $\mathrm{S}$ and let $P_{X}(R)$ denote the probability that the linear order relation takes $R$, i.e., $P_{X}(R)=P r o b(X=R)$. The binary choice probabilities, $f \mathrm{f}_{\mathrm{ij}} ; \mathrm{i}_{i j} \mathrm{j} \mathrm{N} ; \mathrm{i} \in \mathrm{j} \mathrm{j}$ is de...ned as follows.

$$
p_{\mathrm{IJ}}, 0 \text { and } p_{\mathrm{IJ}}+p_{\mathrm{j} i}=1 \text { for every } i ; j 2 \mathrm{~N} \text { with } \mathrm{i} \in=\mathrm{j} \text { : }
$$

The number $p_{i j}$ is interpreted as the probability that $i$ is preferred to $j$. If a probability distribution $X$ is given, the binary choice probabilities $f_{\mathrm{IJ}} ; \mathrm{i}_{i j} 2 \mathrm{~N} ; \mathrm{i} \in \mathrm{j} g$ are consistently determined as follows.

$$
P_{1 j}={ }_{R 2 f Q 2 S j i Q j g} P_{X}(R) \text { for every } i ; j 2 N \text { with } i \quad=j:
$$

The binary choice probabilities are said to be consistent if there exists a probability distribution $X$ that satis...es (2). In this case, we say that the probability distribution $X$ induces the binary choice probabilities $f p_{i j} ; i ; j 2 N ; i \in j g$. If a probability distribution $X$ on $S$ is given, constructing a consistent binary probabilities can be easily performed by equation (2). However, binary choice probabilities are arbitrarily de...ned by (1) without referring to a probability distribution on $\mathrm{S}$. 
Thus, given binary choice probabilities, it is not trivial to determine whether they are consistent. Also not trivial is to recover the probability distribution which induces the given binary choice probabilities when they are consistent.

The binary choice probability problem: When is there a probability distribution $X$ on $S$ that satis...es (2) for a given binary choice probability system $f_{i j}$; $i$; $2 \mathrm{~N}$; i $\in$ jg?

Up to now, several necessary conditions are known for this problem but the su $₫$ cient condition which holds for every $\mathrm{jNj}$ has not been known. While the binary choice probability problem originally dates back to Block and Marschak (1960), recently much attention has been paid again on this problem. As for the motivation, background, history, and current researches of the binary choice probability problem, the readers are referred to Falmagne (1978), Fishburn and Falmagne (1989), Gilboa (1989), Cohen and Falmagne (1990), Marley (1990), and Fishburn (1990, 1992).

\section{I-projection modeling for binary choice probability problem}

While the binary choice probability problem has been de...ned as a problem to ...nd the condition for the existence of a probability distribution that induces given binary choice probabilities, here we formulate it as the 1-projection problem to ...nd the best probability distribution over $\mathrm{S}$ that induces given binary choice probabilities. To ... $x$ the idea, I use the case of $N=f 123 g$. Any binary choice probabilities $\mathrm{fp}_{\mathrm{jJ}} \mathrm{g}$ can be represented by an $\mathrm{jNj} £ \mathrm{jNj}$ table of pairwise comparison data such as Table 1.

Table 1: Binary choice probabilities

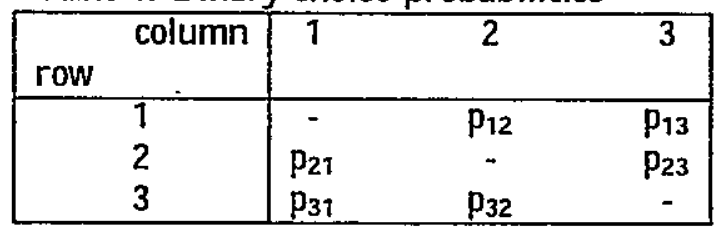

Table 2: Linear orders for cubic case

\begin{tabular}{|cc|ccc|}
\hline Ranking $\mathrm{R}$ & $\mathrm{P}_{\mathrm{X}}(\mathrm{R})$ & 1R2 & 1R3 & 2R3 \\
\hline 123 & $\mathrm{q}_{1}$ & 1 & 1 & 1 \\
132 & $\mathrm{q}_{2}$ & 1 & 1 & 0 \\
213 & $\mathrm{q}_{3}$ & 0 & 1 & 1 \\
231 & $\mathrm{q}_{4}$ & 0 & 0 & 1 \\
312 & $\mathrm{q}_{5}$ & 1 & 0 & 0 \\
321 & $\mathrm{q}_{6}$ & 0 & 0 & 0 \\
\hline & & $\mathrm{p}_{12}$ & $\mathrm{p}_{13}$ & $\mathrm{p}_{23}$ \\
\hline
\end{tabular}

Table 2 lists all linear orders on $N$ and indicates respective truth values of 1R2,1R3, and 2R3 for each linear order $R$. For example, the linear order $R=123$ means that $1 R 2$ and $2 R 3$ hold true, so that by transitivity of $R, 1 R 3$ is also true. These truth values are shown as three 1 's in the last three columns of the ...rst row in the table. Other rows can be interpreted in the same way.

The binary choice probabilities, $p_{12}, p_{13}$, and $p_{23}$ are listed at the bottom line of Table 2 . If they are consistent, equation (2) must hold. The equation (2) means that $p_{i j}$ equals the sum of the probability $P_{x}(R)$ over every $R$ where $i R j$ holds true. Thus the consistency implies that $p_{12} ; p_{13}$, and $p_{23}$ must equal the respective column sums of probabilities $q_{1}$ to $q_{6}$, where summation is taken over every row where truth value is 1 . Hence the consistency condition can be summarized as the following equation (3). Given a probability distribution $f q_{i} g$, it is easy to construct consistent binary choice probabilities $f_{p_{i j}} g$ from (3). Our problem is its inverse. Given the binary choice probabilities $f p_{i j} g$, we must solve the equation (3) for the unknown probability $f q_{k} ; k=1 ; ;: ; 6 g$.

$$
\begin{aligned}
& q_{1}+q_{2}+q_{5}=p_{12} \\
& q_{1}+q_{2}+q_{3}=p_{13} \\
& q_{1}+q_{3}+q_{4}=p_{23}
\end{aligned}
$$

There seem to be many probability distributions on $\mathrm{S}$ that satisfy equation (3). We need some criterion to choose the best. This is just a linear statistical inverse problem. From the axiomatic argument on the logically valid inference for linear inverse problem (Shore, Johnson (1980), Csiszár (1991)), we can employ Kullback-Leibler information as such a criterion. Therefore, the binary choice probability problem can be expressed as the following I-projection problem to ...nd the 
I-projection $q^{\mathbb{n}}$ onto $\mathrm{K}$.

$$
I\left(q^{n} ; y_{4}\right)=\min _{q 2 K} I\left(q ; y_{4}\right)=\min _{f q ; g 2 K} \times \underset{i=1}{q_{i} \log \frac{q_{i}}{y_{4 i}}} \text { ? }
$$

where I $(q ; 1 / 4)$ denotes Kullback-Leibler information of $q$ with respect to $1 / 4, K$ is the convex set of probability distributions satisfying (3), and $y_{4}$ is the given initial probability distribution.

It is instructive to note that the above inverse formulation for binary choice probability problem is closely associated with various interesting topics: image reconstruction, latent class model, maximum likelihood estimation under incomplete data, and decomposability of $(n ; m)$-tournament into chains (Fishburn (1987)). (For further detail, see Saito (1998))

\section{A choice theoretic characterization of Saaty's eigenvector method}

Now the I-projection modeling for binary choice probability problem is applied to a choice theoretic characterization of Saaty's eigenvector method. The essential point of this characterization lies in viewing the outcome reciprocal matrix as ratios of binary choice probabilities since the outcome reciprocal matrix $f_{i j} g$ corresponds to binary choice probabilities $f_{p_{i j}} g$ with one-to-one by $p_{i j}=$ $\frac{a_{11}}{a_{11}+1}$, i.e., $a_{i j}=\frac{p_{i 1}}{p_{11}}$. With this, ...rst the outcome matrix is transformed into binary choice probabilities and then the I-projection modeling is applied to obtain the 1-projection onto the binary choice probability constraint.

1 begin with the simple case of the $2 £ 2$ matrix. Let $A$ denote a positive reciprocal outcome matrix and $\mathrm{P}$ the corresponding binary choice probability matrix.

$$
A=\underset{1=a_{12}}{1} a_{12} \quad P=\frac{1}{\frac{1}{a_{12}+1}} \underset{1}{\frac{a_{12}}{a_{12}+1}} .
$$

A simple calculation shows that the Frobenius root of $A$ is 2 and the corresponding principal eigenvector $w=\left[w ; w_{2}\right]^{0}$ satis...es $a_{12}=w_{1}=w_{2}$. If we normalize the eigenvector $w$ as $w^{0} 1=$ $w_{1}+w_{2}=1$, we see that $w_{1}=a_{12}=\left(a_{12}+1\right)$. Thus the eigenvector $w$ in this case equals the binary choice probability. The I-projection problem to ...nd the I-projection of the uniform distribution onto the binary choice probability constraint is formulated as follows. Let $p_{1}=\operatorname{Pr}(12)$ and $\mathrm{P}_{2}=\operatorname{Pr}(21)$, where $\operatorname{Pr}(12)$, for example, denotes the probability of the ranking 12, i.e., 1 is ranked as the ...rst and 2 as the second. Given $u=[1=2 ; 1=2]$,...nd $p^{a}$ such that

$$
I\left(p^{\mathbb{n}} ; u\right)=\min _{\mathrm{p} Z \mathrm{~K}} I(p ; u)=\min _{\mathrm{p} 2 \mathrm{~K}} p_{1} \log \frac{\mu_{p_{1}}}{u_{1}}+p_{2} \log \frac{\mu_{p_{2}}}{u_{2}} I^{3 / 4} ;
$$

where $K$ is the convex set of probability distributions satisfying constraints (6).

$$
\begin{aligned}
& p_{1}=a_{12}=\left(a_{12}+1\right) \\
& p_{1}+p_{2}=1
\end{aligned}
$$

Apparently, $p_{1}^{u}=a_{12}=\left(a_{12}+1\right)$ so that the l-projection $p^{n}$ in this case is identical to $w$ under any positive initial distribution.

Proposition 1 In the $2 £ 2$ case, the Saaty's eigenvector method and the 1-projection problem under the binary choice probability constraint produce the identical solution.

Next let us consider the case for the $3 £ 3$ positive reciprocal matrix. In the following examples, (A) and $w$ respectively denote the Frobenius root and the corresponding principal eigenvector of $\vec{A}$. The vector $p^{n}$ denotes the I-projection onto the constraints of binary choice probabilities. Its components are lexicographically ordered as follows.

$$
\mathrm{p}^{\mathrm{d}}=[\operatorname{Pr}(123) ; \operatorname{Pr}(132) ; \operatorname{Pr}(213) ; \operatorname{Pr}(231) ; \operatorname{Pr}(312) ; \operatorname{Pr}(321)]:
$$


To compare the eigenvector $w$ and the I-projection $p^{a}$, the 1-projection $p^{\not}$ must be transformed into some comparable ratio scale, say $z$. Here the probability that the $i$-th object is chosen as the top is regarded as its ratio scale $z_{1}$.

$$
z=\left[z_{1} ; z_{2} ; z_{3}\right]=[\operatorname{Pr}(123)+\operatorname{Pr}(132) ; \operatorname{Pr}(213)+\operatorname{Pr}(231) ; \operatorname{Pr}(312)+\operatorname{Pr}(321)]
$$

We show two numerical examples below.

Example 1

$$
\begin{aligned}
& A=\begin{array}{cccc}
2 & 1 & 2 & 7^{3} \\
1=2 & 1 & 5 & 5 \\
1=7 & 1=5 & 1
\end{array} \\
& \text { (A) }=3: 0142 \\
& W=[0: 592 ; 0: 333 ; 0: 075]
\end{aligned}
$$

\section{Example 2}

$$
\begin{aligned}
& A=\begin{array}{lccc}
2 & 1 & 2 & 4 \\
\substack{1=2 \\
1=4} & 1 & 2 & 5 \\
1=2 & 1
\end{array} \\
& \text { (A) }=3 \\
& w=[0: 571 ; 0: 286 ; 0: 143]
\end{aligned}
$$

$$
P=4 \begin{array}{cccc}
2 & 0 & 2=3 & 7=8 \\
1=3 & 0 & 5 \\
1=8 & 1=6 & 0
\end{array}
$$

$p^{\text {" }}=[0: 516 ; 0: 115 ; 0: 243 ; 0: 074 ; 0: 035 ; 0: 016]$

$\mathrm{Z}=[0: 632 ; 0: 317 ; 0: 051]$

$$
\begin{aligned}
& 20 \quad 2=3 \quad 4=5^{3} \\
& P=4 \quad 1=3 \quad 0 \quad 2=35 \\
& 1=5 \quad 1=3 \quad 0 \\
& \mathrm{p}^{\mathrm{A}}=[0: 377 ; 0: 211 ; 0: 211 ; 0: 078 ; 0: 078 ; 0: 044] \\
& \mathrm{z}=[0: 589 ; 0: 290 ; 0: 121]
\end{aligned}
$$

It is interesting to note that solutions to the I-projection problems are close to those of the Saaty's eigenvector method. While they are close to each other, they are diaerent even in this cubic case. It is instructive to see Example 2 because the matrix $A$ is consistent, i.e., rank $A=1$ and $w_{1}: w_{2}: w_{3}=1:(1=2):(1=4)$. Even in this consistent case, the solution to the 1-projection problem is not identical to the principal eigenvector of the consistent matrix. This dixerence waits for some explanation and is worth investigating further here. To say the conclusion in advance, the following theorem states the condition when the solution to the I-projection problem reproduces the Saaty's eigenvector for the consistent case.

Theorem 2 The 1-projection onto the intersection of the binary choice probability constraint (3) and the sequential choice constraints (10) reproduces the Saaty's eigenvector for the $3 £ 3$ consistent reciprocal outcome matrix.

This theorem asserts that the following 1-projection problem (9), in which the sequential choice constraints (10) is added to binary choice probability constraints (3), recovers the Saaty's eigenvevector in the $3 £ 3$ consistent case.

$$
I\left(p^{\mathrm{u}} ; u\right)=\min _{q 2 K} I(q ; u)=\min _{q 2 K} \times q_{i=1} \mu_{i} \log \frac{q_{i}}{u_{i}} ;
$$

where $K$ is a convex set of probability distributions satisfying the following sequential choice constraints (10) in addition to the binary choice probability constraints (3).

$$
\begin{aligned}
& p_{23}\left(q_{1}+q_{2}\right)=q_{7} \\
& p_{13}\left(q_{3}+q_{4}\right)=q_{3} \\
& p_{12}\left(q_{5}+q_{6}\right)=q_{5}
\end{aligned}
$$

In fact, the next example 3 shows that the 1-projection reproduces the Saaty's eigenvector if the additional constraints (10) is added to the previous Example 2.

Example 3 .

$$
\begin{aligned}
& A=\begin{array}{cccc}
2 & 1 & 2 & 4^{3} \\
1=2 & 1 & 2 \\
1=4 & 1=2 & 1
\end{array} \\
& \text { (A) }=3 \\
& \vec{w}=[0: 571 ; 0: 286 ; 0: 143]
\end{aligned}
$$

$$
\begin{aligned}
& 2 \begin{array}{ccc}
0 & 2=3 & 4=5 \\
P= & 4 & 3 \\
1=3 & 0 & 2=3 \\
1=5 & 1=3 & 0
\end{array} \\
P^{u}= & {[0: 381 ; 0: 190 ; 0: 229 ; 0: 057 ; 0: 095 ; 0: 048] } \\
& Z=[0: 571 ; 0: 286 ; 0: 143]
\end{aligned}
$$


To see why this is so, some choice theoretic considerations are needed. For the purpose, ...rst Luce's Theorem must be explained. Let $P_{x}(x)$ denote the choice probability that $x$ is chosen from $X$, i.e., $P_{x}(x), 0$ for all $x 2 x$ and ${ }_{x 2 x} P_{x}(x)=1$. The following condition is the so-called I.I.A. (Independence from Irrelevant Alternatives) condition. This condition is also called the Luce's choice axiom. (Luce and Suppes (1965, p.336)) Under I.I.A. condition, the Luce's theorem holds. (Luce and Suppes (1965, Theorem 31, p.336))

I. l.A. Condition: $A$ set of choice probabilities de...ned for all the subsets of a ...nite set $A$ is said to satisfy the I.I.A. condition if for all $X ; Y$; and $X$ such that $X 2 Y 1 / 2 \times 1 / 2 A$, it holds that $P_{Y}(x)=P_{X}(x j Y)$ whenever the conditional probability exists.

Theorem 3 [Luce's Theorem]. If the I.I.A. condition holds and $A$ is ...nite, then there exists a ratio scale $u(t)$ on $A$ such that for any $\operatorname{PY}_{Y}(x)$ dimerent from 0 or 1 ,

$$
P_{Y}(x)=\frac{P u(x)}{y 2 Y u(y)}
$$

From this theorem, the strict utility model is de...ned as follows.

De...nition 1 [Strict Utility Model]. A set of choice probabilities is said to be a strict utitlity model if there exists a ratio scale $u(t)$ on $A$ such that for all $\times 2 Y y / 2 A$, equation (11) is holds.

Recall that the Saaty's eigenvector method intends to estimate the decision-maker's ratio scale as the principal eigenvector of the positive reciprocal outcome matrix. If we further assume that the estimated ratio scale determines all the conditional choice probabilities by (11), the estimated ratio scale induces a strict utility model. Under this assumption, the Saaty's eigenvector method can be thought of as a method to identify the decision-maker's strict utility model. On the other hand, there is another class of probabilistic choice models called random utility models. Let $A$ be a ...nite set of objects. Let $U(t)$ be a function on $A$ such that, for each $x$ in $A, U(x)$ is a random variable. The vector $U=f U(x): x 2 \mathrm{Ag}$ is called a random vector $U$ on $A$.

De...nition 2 [Random Utility Model]. A set of choice probabilities is said to be a random utility model if there exists a random vector $U$ on $A$ such that for $\times 2 Y y / 2 A$,

$$
\operatorname{Pr}(x)=\operatorname{PrfU}(x)=U(y) ; y 2 Y g:
$$

If the components of the random vector $U$ are independent with each other, the random utility model is said an independent random utility model.

It is well known that any strict utility model is an independent random utility model but the converse is not true. (Luce and Suppes (1965, p.338, Theorem 32)) With respect to the binary choice probability problem, the following theorem due to Block and Marschak (1960) is important. (Luce and Suppes (1965, p.352, Theorem 49)) Let $A=f 1 ; 2 ;: ; ;$ ng and let $S$ denote the set of all $n$ ! linear orders of $A$. Thus $R 2 S$ is a particular ranking of $A$, and $x$ Ry means that $x$ precedes $y$ in $R$. For $x 2 Y y / 2 A$, let $S(x ; Y)=f R 2$ SjxRy for all y $2 Y n f x g g=f R 2$ Sjx precedes y for all y 2 Y nfxg in Rg:

Theorem 4 [Block and Marschak (1960)] A set of choice probabilities $P_{Y}, Y 1 / 2 A$ is a random utility model if and only if there exists a probability distribution $p$ on $S$ such that for $\times 2 Y \frac{1}{2} A$,

$$
\operatorname{Pr}(x)=\underbrace{X}_{\operatorname{R2S}(x ; Y)} p(R):
$$

The solution of I-projection problem under binary choice probability constraints is a probability distribution on $S$ so that if we de...ne the choice probabilities by (13), we obtain a random utility model. Obviously, the solution conforms to given binary probabilities. Thus, by solving the 1 projection problem, we have obtained a random utility model which induces given binary choice probabilities. 
The point is that the strict utility model and the random utility model are diaerent. As to this point, the following theorem also due to Block and Marschak (1960) should be noted. This theorem speci...es the condition that a random utility model becomes a strict utility model and vice versa. (Luce and Suppes (1965, p.354, Theorem 50)) The theorem introduces another familiar notion of sequential choice. A particular ranking can be seen to be obtained from the sequential choice in which the ...rst one is selected among all alternatives, the second is selected as the top among the remaining alternatives, and so forth. Let $A$ and $S$ be as above. For $R 2 S$, let $R_{i}$ denote the element of $A$ that is ranked as the $i$-th. Let $p$ be a probability distribution over $S$ and $P_{Y}(x)$ denote the choice probability that $\times 2 A$ is selected from $Y 1 / 2 A$.

$$
P(R)=P_{A}\left(R_{1}\right) P_{A^{n f R_{1} g}}\left(R_{2}\right)::: P_{f R_{1}} \text { 1;Rng }\left(R_{n_{1} 1}\right):
$$

Theorem 5 [Block and Marschak (1960)] If a set of choice probabilities $P_{Y}, Y \quad 1 / 2 A$ is a strict utility model, then there exists a probability distribution $p$ on $S$ such that $p$ and $P_{y}$ satisfy both equations (13) and (14). Conversely, if there exist $p$ and $P_{Y}$ that satisfy both equations (13) and (14), then the set of choice probabilities $P_{Y}, Y 1 / 2 A$ is a strict utility model.

From this theorem, it is conjectured that the additional sequential choice constraints (14) must be added to the binary choice probability constraints for the I-projection problem to have the same solution as the Saaty's eigenvector. This conjecture is true as stated in Theorem 2. The constraints (10) are derived from equation (14). When $A=f 1 ; 2 ; 3 g$ and $n=3$, the equation (14) is expressed as $p\left(a_{1} a_{2} a_{3}\right)=P_{A}\left(a_{1}\right) P_{A n f a} g\left(a_{2}\right)$ where $a_{1} ; a_{2} ; a_{3} 2 A$. Since $P_{A n f a} g\left(a_{2}\right)$ is equivalent to $\mathrm{P}_{\mathrm{fa}_{2}: \mathrm{a}_{3} \mathrm{~g}}\left(\mathrm{a}_{2}\right)$, it is just a binary choice probability. From this, the ...rst equation in (10), for example, is derived as follows:

$$
\operatorname{Pr}(123)=q_{1}=P_{f 1 ; 2 ; 3 g}(1) P_{f 2 ; 3 g}(2)=(\operatorname{Pr}(123)+\operatorname{Pr}(132)) p_{23}=\left(q_{1}+q_{2}\right) p_{23}:
$$

Now let us consider Example 4. This example shows that the I-projection problem is not always solvable.

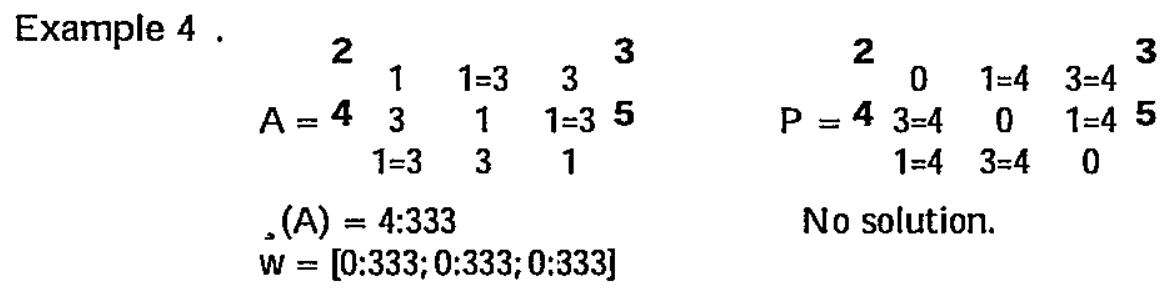

The reason why Example 4 has no solution is closely related to the so-called triangle inequality condition. The triangle inequality for binary choice probabilities $f p_{1 j} g$ is de...ned as follows.

Triangle Inequality: For any distinct $i ; j ; k 2 f 1 ; 2 ;: ; ; n g ;$

$$
p_{i j}+p_{j k}+p_{k i} \cdot 2:
$$

In the present $3 \pm 3$ case, it is easy to check that the triangle inequality is the necessary and suథcient for the binary choice probability problem to have a solution. In the above Example 4 , the triangle inequality does not hold, i.e., $p_{13}+p_{32}+p_{21}=3=4+3=4+3=4=9=4>2$ : Now I give the proof of Theorem 2.

Proof: Let $w_{1}$ denote the ratio scale for the $i$-th object, whose sum is normalized to 1 , i.e., $w_{1}+w_{2}+w_{3}=1$. Since the consistent reciprocal outcome matrix $\left(a_{i j}\right)$ satis...es $a_{i j}=\frac{w_{1}}{w_{j}}$, binary choice probabilities are expressed as $p_{i j}=\frac{w_{i}}{w_{i}+w_{j}}$. Solving equation (3) of binary choice probability constraints for $q_{1} ; q_{2} ; q_{3} ; q_{6}$ gives the following equation (16).

$$
\begin{aligned}
& q_{1}^{\prime}=p_{12}+p_{23} ; p_{13} ; q_{4} i q_{5} \\
& q_{2}=p_{13} ; p_{23}+q_{4} \\
& q_{3}=p_{13} ; p_{12}+q_{5} \\
& q_{6}=1 ; p_{13} ; q_{4} ; q_{5}
\end{aligned}
$$


Substituting (16) into (9), the ...rst order condition for I-projection problem in the cubic case is obtained as follows.

$$
\begin{aligned}
& { }^{3} q_{2} q_{4} \\
& \log _{3} \frac{u_{1} u_{6} q_{6}}{u_{2} u_{4}}=0: \\
& \log \frac{q_{3} q_{5}}{q_{1} q_{6}}\left(\frac{u_{1} u_{6}}{u_{3} u_{5}}=0:\right.
\end{aligned}
$$

From $\frac{p_{11}}{P_{j i}}=\frac{w_{1}}{w_{1}}$, the sequential choice constraints (10) become equivalent to $\frac{\operatorname{Pr}(i j k)}{\operatorname{Pr}(i k j)}=\frac{w_{1}}{w_{k}}$, i.e., $\frac{g_{1}}{q_{2}}=$ $\frac{w_{2}}{w_{3}} ; \frac{q_{3}}{q_{4}}=\frac{w_{1}}{w_{3}}$, and $\frac{q_{5}}{q_{6}}=\frac{w_{1}}{w_{2}}$. Substituting these expressions for $q_{4}$ and $q_{5}$ into (16) and solving for $q_{6}$ gives $q_{6}=\frac{w_{3}}{w_{2}+w_{3}}$. Then $q_{5}=\frac{w_{1} w_{3}}{w_{1}+w_{2}}$. Thus $q_{5}+q_{6}=w_{3}$. Similarly, $q_{i}+q_{2}=w_{1}$ and $q_{3}+q_{4}=w_{2}$ hold. Note that the ...rst order condition (17) has not been used. Hence if the given binary choice probablties satisfy the triangle inequality, binary choice and sequential choice constraints uniquely determine the I-projection. Q.E.D.

\section{A choice theoretic validation for Saaty's eigenvector method}

A key point of Saaty's eigenvector method is the presumption that the inconsistency is captured by the residual matrix $E=\left("{ }_{i j}\right)=\left(a_{i j} w_{j}=w_{i}\right)$, where the matrix $A=\left(a_{i j}\right)$ is the outcome positive reciprocal matrix and $w=\left(w_{i}\right)$ is the principal eigenvector. Here I discuss this topic on the inconsistency represented by the residual error terms in Saaty's method. To tell the truth, the matrix A in Example 4 is a typical instance of the residual matrix for Saaty's eigenvector method. This is known from the following proposition due to DeTurck (1987). Saito (1998) gives its proof from the viewpoint of permutation group. This proposition also is deeply related to the necessary and suథcient condition that the right and the left eigenvector are mutually reciprocal for a positive reciprocal matrix. For details, see Saito (1998).

Proposition 6 In the $3 £ 3$ case, the only possible structure of the residual matrix is the following.

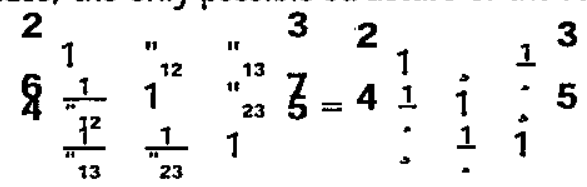

The essential point of Saaty's eigenvector method is that the residual matrix contributes nothing to the estimated ratio scale. This is because the residual matrix equals the scalar multiplication of,$(A)$ and a stochastic reciprocal matrix so that its right eigenvector is proportional to 1 . Furthermore, in the $3 \mathrm{E} 3$ case, its residual becomes a scalar multiple of reciprocal doubly stochastic matrix as seen from the above theorem so that the left eigenvector also is proportional to 1 . This gives rise to a question: Does the I-projection problem for the residual matrix reproduce the same ratio scale as proportional to 1 ? The next example gives an ađrmative answer to this question. The matrix A in Example 5 is the residual matrix for Example 1.

Example 5

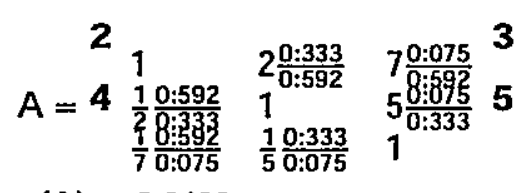

$$
\begin{aligned}
& (A)=3: 0138 \\
& w=[0: 333 ; 0: 333 ; 0: 333]
\end{aligned}
$$

3

5

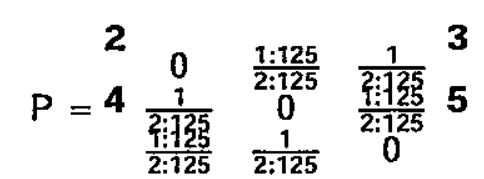

$p^{\mathrm{n}}=[0: 196 ; 0: 137 ; 0: 137 ; 0: 196 ; 0: 196 ; 0: 137]$

$\mathrm{z}=[0: 333 ; 0: 333 ; 0: 333]$

This result holds for the solvable $3 £ 3$ case. I have not known whether this is true even in the case of higher dimensions greater than 3 . Though limited only to a $3 \notin 3$ case, this fact gives another theoretical validation for Saaty's eigenvector method from the choice theoretic perspective. I state this fact as a theorem.

Theorem 7 [Saito (1998)] Consider the $3 £ 3$ residual reciprocal matrix with the form (18). De...ne binary choice probabilities $f p_{i j}: i 6=j g, 1 \cdot i_{i j} \cdot 3$ with $p_{i j}+p_{j i}=1$ by

$$
p_{12}=p_{23}=\frac{s}{s+1} ; p_{13}=\frac{1}{s+1}:
$$


Suppose $2 p_{12}, p_{13}$. Then, the solution $q^{n}$ of the I-projection problem with the initial uniform probability distribution under the binary choice probability constraints (19) is given as follows. For $1=3 \cdot \mathrm{P}_{12} \cdot 2=3$,

$$
\begin{aligned}
& q_{1}^{\mathrm{u}}(123)=p_{12} i \frac{1}{3} ; q_{2}^{\mathrm{u}}(132)=\frac{2}{3} i p_{12} ; \\
& q_{3}^{\mathrm{u}}(213)=\frac{2}{3} i p_{12} ; q_{4}^{\mathrm{u}}(231)=p_{12} i \frac{1}{3} ; \\
& q_{5}^{\mathrm{a}}(312)=p_{12} i \frac{1}{3} ; q_{6}^{\mathrm{u}}(321)=\frac{2}{3} i p_{12}:
\end{aligned}
$$

The probability that each alternative is selected as a top ranked is $\frac{1}{3}$. Furthermore, the probability that each alternative is chosen as the second and as the last also are $\frac{1}{3}$.

Proof. The optimal conditions for the I-projection problem are (16) and (17). From (16) and (17), we have

$$
\begin{aligned}
& q_{1}=2 p_{12} i p_{13} i \frac{q_{1} q_{6}}{q_{2}} i \frac{q_{1} q_{6}}{q_{3}} ; q_{2}=p_{13} i p_{12}+\frac{q_{1} q_{6}}{q_{2}} ; \\
& q_{3}=p_{13} i p_{12}+\frac{q_{1} q_{6}}{q_{3}} ; q_{6}=1 ; p_{13} i \frac{q_{1} q_{6}}{q_{2}} i \frac{q_{1} q_{6}}{q_{3}}:
\end{aligned}
$$

From the second and the third equations, we get $q_{2}=q_{3}$. With $q_{2}=q_{3}$, the ...rst and the last equations give $q_{1}=p_{13} \mid 2 q_{2}$; and $q_{6}=1+p_{13} ; 2 p_{12} \mid 2 q_{2}$ : Substituting these into the second equation leads to $3 q_{2}^{2} i\left(3\left(p_{13} \mid p_{12}\right)+2\right) q_{2}+p_{13}\left(1+p_{13} \mid 2 p_{12}\right)=0$. This is equivalent to $\left(q_{1}+q_{2}\right)\left(3\left(q_{1}+q_{2}\right) ; 1\right)=0$ : A simple calculation derives (20). Q.E.D.

The above result implies that no "partial" information is contained in the residual matrix in the sense that I-projection solution for the residual makes no "partial" contribution to the probability how each object is ranked among three. Hence Theorem 7 gives another choice theoretic validation for Saaty's eigenvector method.

Theorem 7 also says that if $2 p_{12}, p_{13}$, the solution for $1=3 \cdot p_{12} \cdot 2=3$ is given by (20). In terms of , this means $1=2$., 2 . If,$<1=2$, the 1-projection problem has no solution. While the Saaty's eigenvector method interprets the residual matrix as some inconsistency in the pairwise comparison process, this inconsistency may be accrued to two causes: (i) the probabilistic choice of $n$ ! linear orders which are individually consistent; (2) the existence of cycles or nontransitive preferences. We may regard the range $1=2, \ldots, 2$ as a threshold of inconsistency that can be captured by the probabilistic mixture of consistent linear orders. Hence we can consider that the inconsistency with the range,$<1=2$ should be captured by the existence of nontransitive or cyclic preferences.

This interpretation suggests the introduction of cycles or nontransitive preferences into the previous 1-projection problem under binary choice probability constraints. Thus we are led to drop the axiom of transitivity in the linear order $R$. This is equivalent to assuming that (i) $\gg x R x$ for every $x 2 R$ and (ii) for every $x ; y 2 R$, either one of $x R y$ or $y R x$ holds. In the $3 £ 3$ case, we can add two cycles, (123) and (132) to 3 ! linear transitive orders. Hence the I-projection problem is now to obtain the probability distribution not on 3 ! linear transitive orders but on $8(=3 !+2)$ order relations.

In the next Example 6, the two cycles, (123) and (132) are included in the 1-projection problem for the previous Example 4 under the constraints of three binary choice probabilities, $p_{12} ; p_{13}$, and $\mathrm{p}_{23}$. Note that Example 4 is unsolvable without introducing cycles. Here the last two components of $\mathrm{p}^{\beth}$ correspond respectively to $\operatorname{Pr}[(123)]$ and $\operatorname{Pr}[(132)]$. The components of vector $v$ denote the respective probabilities of linear order and cycles. 
Example 6

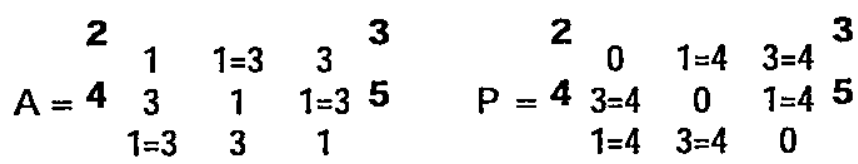

$$
\begin{aligned}
& \text { (A) }=4: 333 \quad p^{\mathrm{u}}=[0: 047 ; 0: 141 ; 0: 141 ; 0: 047 ; 0: 047 ; 0: 141 ; 0: 016 ; 0: 422] \\
& \dot{w}=[0: 333 ; 0: 333 ; 0: 333] \quad z=[0: 188 ; 0: 188 ; 0: 188] \\
& v=[\text { linear; cycle }]=[0: 562 ; 0: 438]
\end{aligned}
$$

Surprisingly, the conditional probability that each alternative is selected as a top among 3 ! linear orders still turns out to be the uniform distribution. This topic is worth investigating further.

\section{References}

Block, H. D. and Marschak, J. (1960). Random orderings and stochastic theories of responses. Reproduced in Economic Information, Decision, and Prediction: Vol. I. by Marschak, J. (1974). pp.172-217. D. Reidel Publishing Co.

Cohen, M. and Falmagne, J. C. (1990). Random utility representation of binary choice probabilities: A new class of necessary conditions. Journal of Mathematical Psychology 34, 88-94.

DeTurck, D. M. (1987). The approach to consistency in the analytic hierarchy process. Mathematical Modelling 9, 345-352.

Csiszár, I. (1991). Why least square and maximum entropy? An axiomatic approach inference for linear inverse problems. The Annals of Statistics 19 2032-2066.

Falmagne, J. C. (1978). A representation theorem for ...nite random scale systems. Journal of Mathematical Psychology 18, 52-72.

Fishburn, P. C. (1987). Decomposing weighted digraphs into sums of chains. Discrete Applied Mathematics 16, 223-238.

Fishburn, P. C. (1990). Binary probabilities induced by rankings. SIAM Journal of Discrete Mathematics 3, 478-488.

Fishburn, P. C. (1992). Induced binary probabilities and linear ordering polytope: A status report. Mathematical Social Science 23, 67-80.

Fishburn, P. C. and Falmagne, J. C. (1989). Binary choice probabilities and rankings. Economic Letters 31, 113-117.

Gilboa, 1. (1989). A necessary but insu $₫$ cient condition for the stochastic binary choice problem. Discussion paper No. 818, The Center for Mathematical Studies in Economics and Management Science, Northwestern University.

Luce, R. D. and Suppes, P. (1965). Preference, Utility, and Subjective Probability. Chapter 19 in Luce, R. E., Bush, R. R., and Galanter, E. (eds.) Handbook of Mathematical Psychology vol 3: 249-410, John Wiley and Sons

Marley, A. A. (1990). A historical and contemporary perspective on random scale representations of choice probabilities and reaction times in the context of Cohen and Falmagne's results. Journal of Mathematical Psychology 34, 81-87.

Saaty, T. L. (1980). The Analytic Hierarchy Process, McGraw-Hill

Saito, S. (1989). Ratio scale decomposition analysis of group preference. Paper presented at TIMS XXIX held at Osaka. Discussion paper No. 7, Faculty of Economics, Fukuoka University.

Saito, S. (1998). Extensions of Iterative Proportional Fitting Procedure and I-projection Modeling. Kyushu University Press. Fukuoka

Shore, J. E. and Johnson, R. W. (1980). Axiomatic derivation of the principle of maximum entropy and the principle of minimum cross-entropy. IEEE Transactions on Information Theory IT-26 26-37. [Correction. (1983). IT-29 942-943]

Vargas, L. G. (1983). Analysis of sensitivity of reciprocal matrices. Applied Mathematics and Computation 12: 301-320. 